\title{
Application of BIM Technology in Interference Check in Construction
}

\author{
Work \\ Liyuan Wang1, a Binjie Qian ${ }^{1, b}$, Xiaotong Cui ${ }^{1, c}$, Liu Chao *1, d \\ ${ }^{1}$ School of Environmental and Municipal Engineering, Qingdao University of Technology, Qingdao, \\ Shandong, 266033,China \\ a838979848@qq.com, b694617746@qq.com, ‘365319610qq.com, dliuchao.hy@163.com
}

Key words: Building Information Modeling; Interference Check; Simulation software

Abstract: As the size, shape and function of construction projects are becoming complex, building system designs are more challenging. The traditional building system design pattern has been unable to meet the current requirement of the overall design of the building. For designers by conventional two-dimensional graphic design, it is difficult to find the space interference between pipes, structures and buildings and to make reasonable adjustments. Using simulation software Revit and Navisworks for Building Information Modeling projects, designers raised a new solution for the cross-cutting issue. In this paper, we take a comprehensive building construction for example to study interference check.

With the development of computer software and hardware, the brand-new design and management pattern with the engineering digital model as the core is gradually emerging. As a result, some people propose BIM concept. BIM technology plays an important role in driving the building industry and BIM is developing rapidly.

\section{Meaning of BIM technology}

BIM indicates "building information model", which regards basic components in the building work as design elements. BIM technology organizes geometric data, physical features and material information of components seamlessly to form a database system with comprehensive building information. All parameters of components will be stored into this database, which is the basis for the data model of a construction project. To meet work requirements, all participants will insert, extract, edit and update information in the model database. The parameter information of different components in the model is not independent information individual. They have certain space and logic relation with each other and compose a complete and hierarchical building information system [1].

\section{Features of BIM technology}

The collaborative work environment based on BIM is sustainable and transparent. Component information of projects can be copied and audited, which ensures that participants communicate with each other timely and share information with each other accurately in the whole lifecycle of projects. To make project managed better, project participants can conduct scientific analysis on the design information shared by different specialties when making a decision. The 3D design process based on BIM technology compared to the traditional design methods, has the following prominent features: 


\section{Visual design}

First, the distinct feature of BIM is what you see is what you get—visual design, which is very meaningful for different building specialties. With increasing complexity of building scale, forms and functions, building systems become more complex. Complex pipes of different systems will be frequently deployed within a limited space to meet corresponding space requirements. BIM can realize visual design, and help designers to intuitively know space conditions of key parts so that designers can deploy corresponding pipes more reasonably, utilize the limited space better, and improve design efficiency and design quality effectively.

Space interference check

In the past, interference check consumed lots of manpower and time. To find a space interference point on the plane drawing, participants should have strong abilities of space imagination. A large number of manpower and time is consumed, however, only important parts are checked and the whole project can not be managed in a macro manner. If the BIM technology is used for design, the space relation of the pipes, buildings and structures of main part can be intuitively observed from different views. And participants can find the space interference between pipes, structures and buildings and make reasonable adjustments [2].

Associated change

The BIM model is a digital file integrating the design information of different specialties. The design information from different specialties will be finally integrated into a model via the collaborative design platform in the design based on BIM. When any professional design information changes, it can be changed in this model. Although this method cannot reduce the amount of change, it can enhance change association and reduce manpower and time.

\section{Collaborative design}

The BIM model is a collaborative design of different building specialties. First, the main files of different specialties can be designed independently. Designers of different specialties can complete design of the main files of respective specialties on their conventional design interface. Then the project files of different specialties can be shared in a link model manner via the collaborative design platform. The model system linking different specialties will be automatically updated with the update of the main files of different specialties, which ensures accurate delivery of design information between different specialties.

\section{Specific application of BIM technology in interference check of building work}

\section{Type of interference check in BIM technology}

The BIM technology which features 3D visualization can be used to check early interference. Then it can optimize work designs, net spaces and pipeline layouts. It can also reduce possible error loss and reworks in the stage of construction. By the 3D interference-optimized pipeline scheme, constructors can carry out construction managements and simulations to improve qualities of construction and capabilities of communication with the owner [3].

The purpose of interference check is to find and report interference between different parts in the project in advance. Interference is divided into hard clash and soft clash (gap clash).

Hard clash

Hard clash: entities are intersected in the space. This clash type is very frequent in the building design, especially when different specialties have no uniform standard height, which occurs among structural beams, air-conditioner pipelines and water drainage and supply pipelines.

Space clash

Space clash: the entity 1 and entity 2 are not intersected in the space, but the space $\mathrm{d}$ between 
two entities is less than the setting tolerance T, so it is regarded as clash. This clash is checked for safety. E.g. the water heat pipeline should have the minimal space with the electric bridge and busbar. The minimal space can be set for different specialties to check if the minimal space meets the design requirement and if the pipeline device covers the sockets and switches on the wall.

\section{Implementation steps of interference check based on BIM technology}

This paper studies a complex building project. In this project, the pipeline system for water supply and drainage includes water supply, water drainage, waste water and fire control system. When the pipelines are integrated, many pipelines are deployed at corridor ceilings or device rooms. When plentiful pipelines are deployed in the limited space, the pipes will clash and intersect with each other inevitably. Error of designers may lead to "hard clash" between the pipes, structural columns and beams, or "soft clash" due to unqualified space between building walls.

The Autodesk Revit MEP software is used to construct the BIM model of the pipeline work of the water supply and drainage system in this project. Based on $3 \mathrm{D}$ simulation model, the interference is checked in the comprehensive pipeline design by using the interference check function of this software and Navisworks software. The main work is divided into four phases [4-5]:

Complete data collection and framework of construction technology

Designers collect the specific data of the building construction, and construct BIM technology framework, and then achieve the data interface and data interaction. The database technology is used to store building construction data and establish the platform layer. The data platform layer includes the data integration management platform and 4D visual platform. It accomplishes data reading, saving, integration and validation function, and constructs sub-information models. Based on different sub-information models, the data platform layer provides data support for the model layer and the application layer. The application layer, the final layer of the BIM technical framework, mainly achieves dynamic project management and interference analysis, provides network progress and resource optimization, and completes modeling [6].

Modeling

Before the BIM model of the water supply and drainage pipeline system is created, the pipelines and pipes should be loaded to the software for parameter editing. By reasonably setting these component parameters, it can reduce later adjustments of pipelines and improve design efficiencies. Designers can define the name of the pipe type to distinguish the pipe type and set properties of the roughness of the segment, materials and connection types, shown as the figure 1.

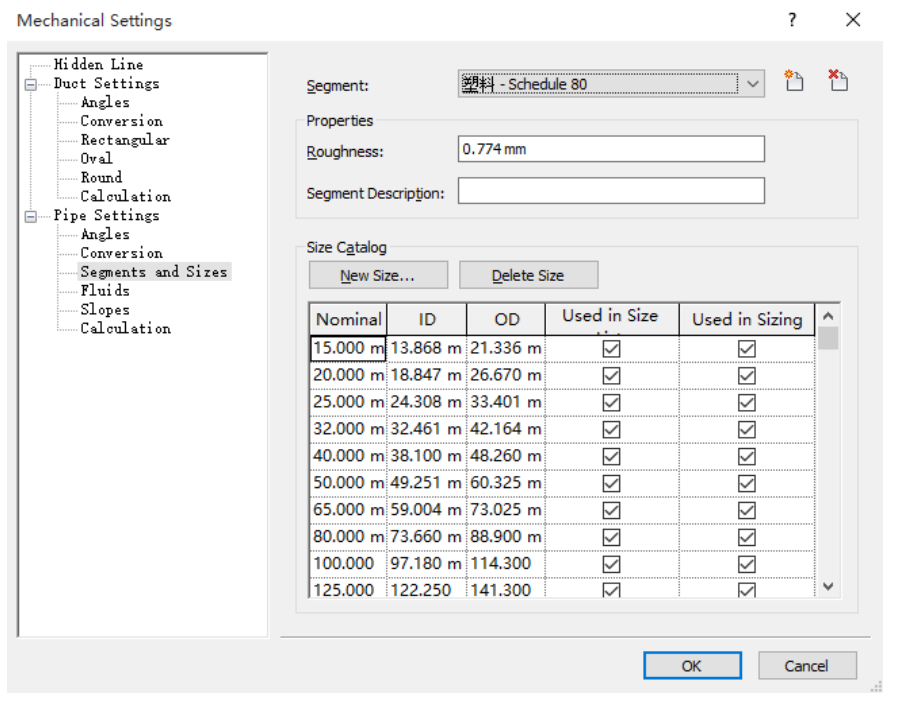

Figure 1 Mechanical Settings

Finishing setting, we create the BIM model of the water supply and drainage pipeline system 
by the software. In addition, to deploy the pipeline system accurately, the software can link the 2D CAD drawing to the project as external reference. Designers can identify the space position of the pipes by referring to the CAD drawing and import it to create a new BIM model. Because of this advantage, we can view CAD drawing on the software design interface directly and avoid possible errors easily [7].

Pipeline integration and interference check

After the 3D simulation software Revit Architecture and MEP 2016 are used to complete BIM model of the building main body and the water supply and drainage pipeline system in the project via collaborative design of different specialties, water supply and drainage professional pipelines should be integrated to find possible interference between pipelines and devices and adjust them[3-4]. Generally after all pipelines are drawn and devices are placed, pipelines should be checked and adjusted roughly in the 3D view. Designers should find the obvious pipeline interference in 3D drawing and adjust the pipeline position by adjusting the standard height. This paper creates the BIM model integrating the building, structural beam, columns and water supply and drainage pipeline information via the collaborative design. The model interference check will focus on interference between pipeline subsystems in the water supply and drainage system of the building as well as interference between main structural components and building's main body.

1) Revit "interference check"

The Autodesk Revit MEP 2016 software can be used to check interference for the BIM mode created in the collaborative design. The interference check function of the software can accurately locate corresponding positions according to the interference report generated by the software and directly change invalid pipes in the model, which is easy to implement [8].

"Interference check" function is shown as the figure 2. A user can select the type of the drawing element and categories in the interference check dialog. The type of the drawing element can be from the current project or linked Revit model. The software can check interference between components in the current project and it can also do between a current project and a linking model in interference check. However, it cannot check interference between two linking models in the project.

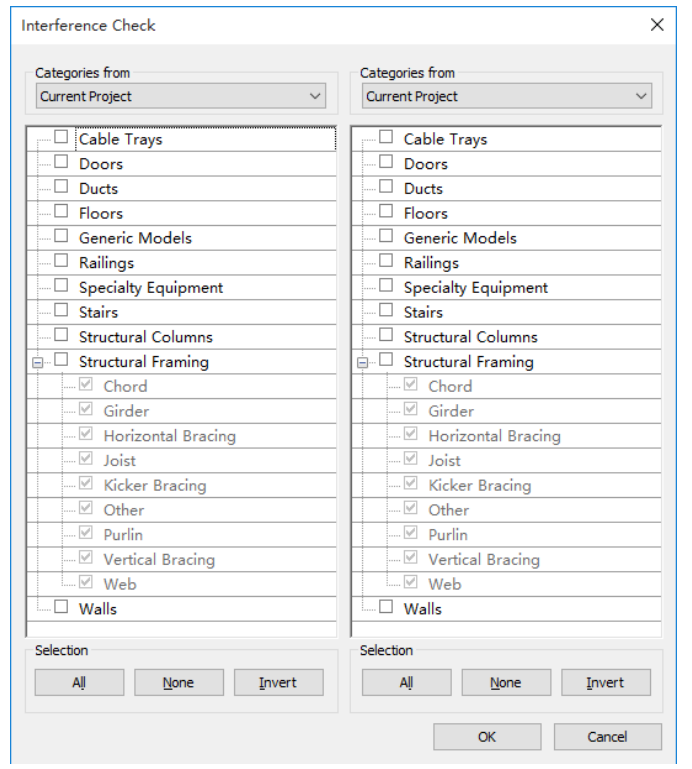

Figure 2 Interference check between current projects

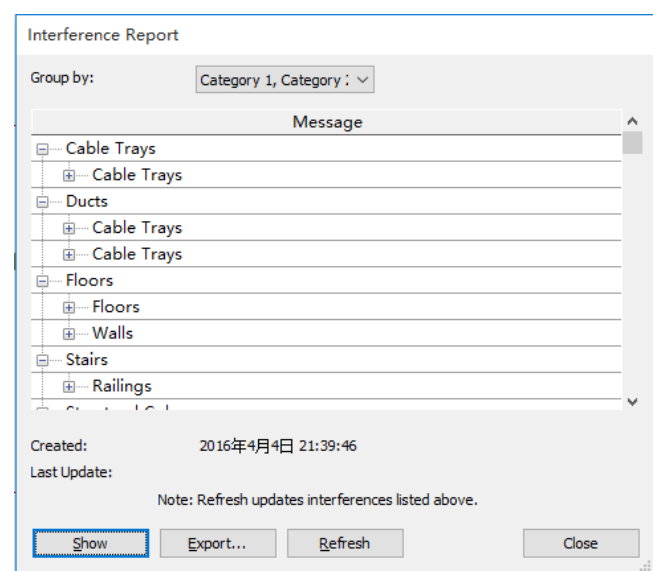

Figure 3 Interference report

After interference check, if no invalid elements, a dialog displays informing you of this. If invalid elements are reported, the software will list all conflict drawing elements and generate the 
interference report shown as the figure 3 . When selecting any conflict message in the interference report, the software will locate the interference object automatically and highlight the interference object. Designers can change drawing elements on the view directly to solve the confliction. Designers can export and save HTML report. After the checked interference is solved, you should refresh the interference report and the solved conflictions will not be displayed.

2) Navisworks "Clash Detective"

The Navisworks software is a special clash detective software and can be compatible to multiple model formats. Using the BIM model of the water drainage pipeline system of the water supply and drainage system and BIM model of the building main system, this paper performs clash detective via the following steps:

(1) Attach files: export nwc files of structural components in water drainage pipelines and the established BIM model by the Autodesk Revit MEP 2016 software and attach exported nwc files to Navisworks software.

(2) Set confliction: Clicking "clashdetective" button in the Navisworks, a user can enter the editing interface. A user should first select objects for the clash test. Structural components (walls, beams and columns) in the water drainage pipeline system and model are selected as confliction check objects in this paper. A user can set type of clash to test in the software. Here we select "hard clash" (namely touch interference between pipes or between pipes and structures). After above settings are completed on the editing window, a user can run clash detective to get the clash report. For detailed settings are referred to the figure 4:

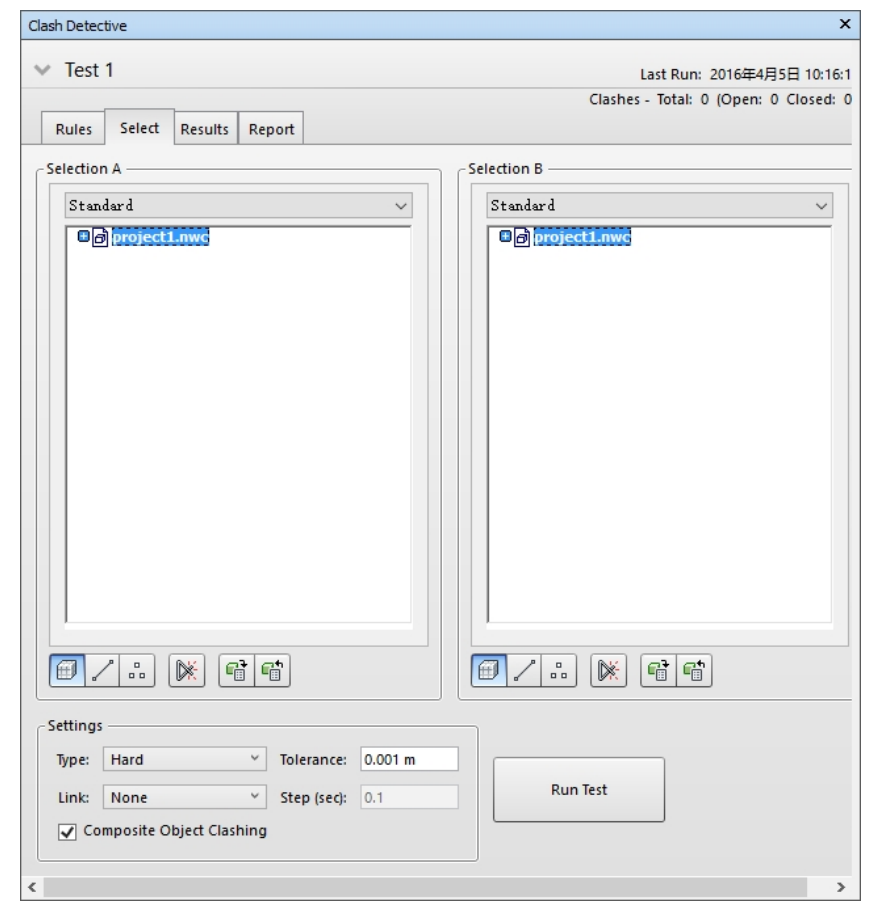

Figure 4 Select tab of clash detective

(3) Identify the clash point information according to the clash report. After running the clash test, the Navisworks software will generate the report automatically. The report will list positions and quantities of clash points. The report will give the ID of clash points and locate clash points by ID number, which is shown as the figure 5 . 


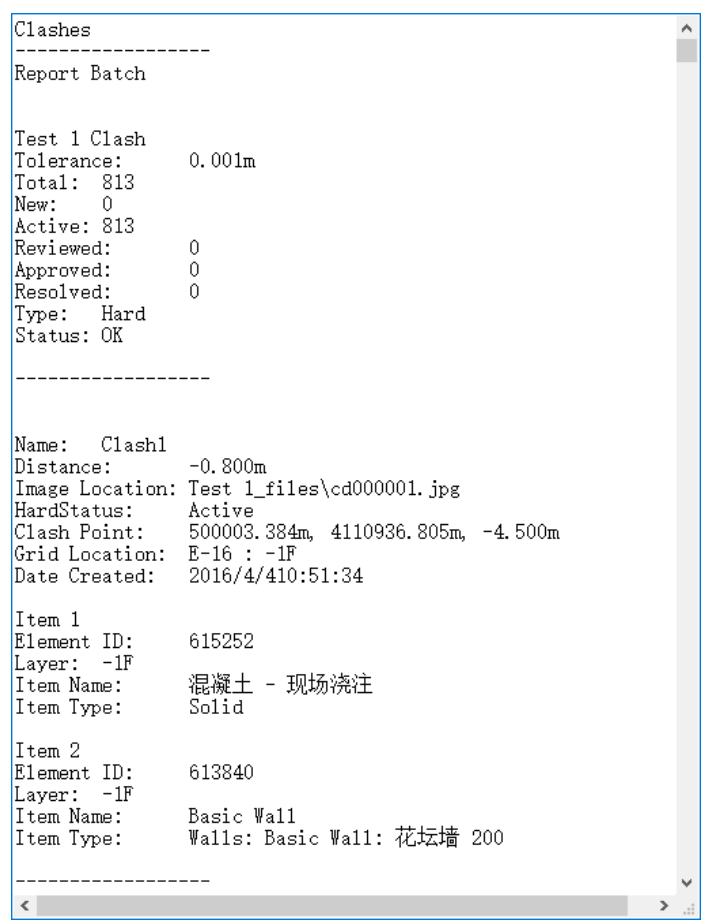

Figure 5 Clash report

Solve problems according to the check report

If intersected interference problems are solved on the design phase, we can reduce redoing and change on the construction phase and avoid waste manpower, material resource and in time due to design errors. After intersected interference of pipelines are found, space positions of crossing pipelines should be reasonably adjusted according to certain rule. A designer should flexibly apply this function in adjustment. Some general rules for pipeline adjustment are described as follows: big pipelines are superior to small pipelines; gravity self-flow pipelines are superior to pressure pipelines; long-term pipelines are superior to short-term pipelines; water drainage pipelines are superior to cooling water pipelines; high-pressure pipelines are superior to low-pressure pipelines. Generally the interference check will be performed several times with changes of the model. The general flow for interference check is shown as the figure 6.

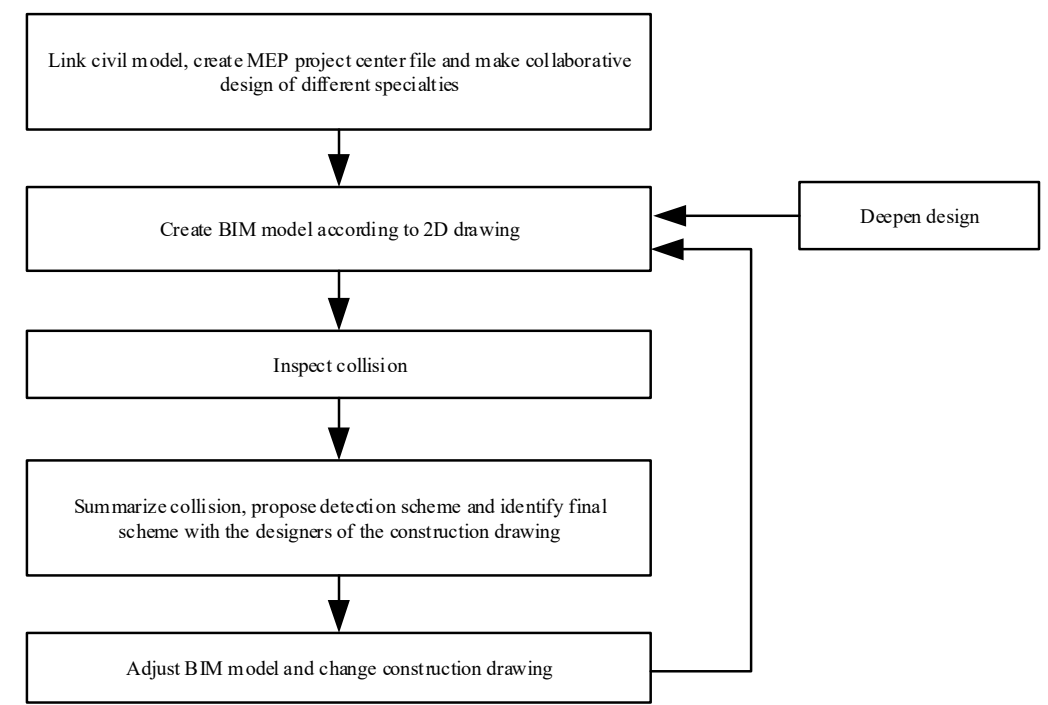

Figure 6 General flow of interference check 


\section{Existing problems in interference check based on BIM technology}

When the water supply and drainage pipeline system is deployed in building work, some software problems may affect the construction efficiency of the model. A designer cannot complete the design in time due to these problems. In addition, the delivery and the quality of final design achievements will be affected.

Components meeting China building design standard are lack and the family libraries are not perfect

The BIM model based on IFC standard (Industry Foundation Classes, which is a data model standard) constructed by Revit software is superior to 3D models constructed by other 3D simulation software, but international standards do not adapt the current building industry of China and have some differences from the domestic standards. Revit software is a foreign software, so its application will be affected by the building standards in China. The standard component family libraries of the software are lack of the families meeting the product standards in China, so standardized family library documents should be expanded in the software and family documents suitable for application in China should be available according to the general market standard in China.

Interference check based on Revit and Navisworks software is not perfect

For a complicated model, the interference check function of Revit is directly used to check interference inside the model. High performance computes are required and a large number of crossing problems may be checked at same time, but it is not easy for a designer to change. Revit can only check "hard clash" and cannot check "soft clash" inside the model, as a result of which it will miss the interference information. For this problem, on the one hand, the model can be subdivided (the whole model is divided by the floor) and interference check is performed separately for the model in different areas. On the other hand, the model can be imported to the Navisworks software. We can roam the model, find obvious problems, change them, and detect clash of the whole model.

The Navisworks software is used to detect clash in the model, but it can only detect clash of different specialty sets of the model, rather than the whole model against itself. For this problem, a single object clash can be checked via Revit API second development programming. As an auxiliary tool for Navisworks clash analysis, it can optimize model clash analysis.

\section{Conclusions}

With the application of BIM technology, the BIM-based multi-specialty collaborative design pattern will become a future trend. Interference check based on Revit and Navisworks software not only provides $3 \mathrm{D}$ design interface for designers, but also realizes easy, convenient and accurate interference check. With the help of BIM, designers can spend more time and energy in professional design and improve qualities of collaborative designs and building projects.

\section{References:}

[1] Qin Jun. BIM Technology in the Building Design [J]. Architecture Technique, 2011 (Z1) :160-163.

[2] Zhang Jianping. Research and application of BIM technology [J]. Construction Technology, 2011 (2):116 119. 
[3] Wang Jun. Study on the application of BIM principle and BIM software in construction projects [D]. Chengdu: SouthWest JiaoTong University, 2011.

[4] Yang Ke, Kang Dengze, Che Chuanbo. BIM-based interference checking in multidisciplinary coordination design research [J]. Journal of Information Technology in Civil Engineering and Architecture, 2013, 5 (4):72-75.

[5] Zhao Honghong, Li Jiancheng. Information Building Design--- Autodesk Revit [M], Beijing: China Building Industry Press, 2005.

[6] Patrick J.O’Connor, Jr. Integrated project delivery: collaboration through new contract forms [M] .2009.

[7] James F.Hoburg. Modeling Maglev Passenger Compartment Static Magnetic Fields From Linear Halfback Permanent-Magnet Arrays, IEEE TRANSACTIONS ON MAGNETICS, VOL.40, N0.1, JANUARY 2004.

[8] Gu Hailing, Gui Tanchun. Application of BIM technology in water supply and drainage design of Shanghai Center Building [J]. Water \& Wastewater Engineering. 2012， 48 (11):92-97. 